\title{
Modelling Heterogeneity among Experts in Multi-criteria Group Decision Making Problems
}

\author{
Ignacio J. Pérez ${ }^{1}$, Sergio Alonso ${ }^{2}$, Francisco J. Cabrerizo ${ }^{3}$, \\ Jie $\mathrm{Lu}^{4}$, and Enrique Herrera-Viedma ${ }^{1}$
}

1 Dept. of Computer Science and Artificial Intelligence, University of Granada, Spain

ijperez@decsai.ugr.es, viedma@decsai.ugr.es

${ }^{2}$ Dept. of Software Engineering, University of Granada, Spain zerjioiaugr.es

3 Dept. of Software Engineering and Computer Systems, Distance Learning University of Spain (UNED), Madrid, Spain

cabrerizo@issi.uned.es

4 Faculty of Information Technology, University of Technology, Sydney, Australia jieluait.uts.edu.au

\begin{abstract}
Heterogeneity in group decision making problems has been recently studied in the literature. Some instances of these studies include the use of heterogeneous preference representation structures, heterogeneous preference representation domains and heterogeneous importance degrees. On this last heterogeneity level, the importance degrees are associated to the experts regardless of what is being assessed by them, and these degrees are fixed through the problem. However, there are some situations in which the experts' importance degrees do not depend only on the expert. Sometimes we can find sets of heterogeneously specialized experts, that is, experts whose knowledge level is higher on some alternatives and criteria than it is on any others. Consequently, their importance degree should be established in accordance with what is being assessed. Thus, there is still a gap on heterogeneous group decision making frameworks to be studied. We propose a new fuzzy linguistic multi-criteria group decision making model which considers different importance degrees for each expert depending not only on the alternatives but also on the criterion which is taken into account to evaluate them.
\end{abstract}

keywords: Group decision making, multi-criteria decision making, heterogeneous decision frameworks, linguistic approach.

\section{Introduction}

Decision making is as very common activity all over the world. Usually, it is performed by people who have to consider some criteria in order to derive the best option from a feasible set. But sometimes, alternatives and criteria are imprecise, contradictory or belong to a wide range. In this case an expert can not make a decision on his own and it is necessary that a group of experts, with a high collective knowledge level on these particular criteria, take part in the decision process. Thus, we interpret the decision process in the framework of group decision making (GDM). 
GDM models are used to obtain the best solution(s) for a problem according to the information provided by some decision makers. Usually, each decision maker (expert) may approach the decision process from a different angle, but they have a common interest in reaching an agreement on taking the best decision. Concretely, in a GDM problem we have a set of different alternatives to solve the problem and a set of experts which are usually required to provide their preferences about the alternatives [1|2 3 4 4 ].

Furthermore, there are GDM problems in which, to evaluate the alternatives, the experts have to take into account the value of some criteria that define the features of each alternative. Multi-criteria decision making (MCDM) refers to making a decision (e.g., evaluation, prioritization, and selection) over the available alternatives that are characterized by multiple, usually conflicting, criteria [6]. In such decision situation the aim is to find a set of alternatives that, considering all the criteria, solves the problem in the best way. Multi-criteria group decision making (MCGDM), which combines MCDM and GDM methods, has been proved to be a very effective technique to increase the level of overall satisfaction for the final decision across the group and particularly in evaluation decision-making such as evaluating products, developing policies, selecting employees, and arranging various resources [7 8 |9 910 |11||12|13|14].

Due to the wide range of different problems that can be solved with GDM models, in recent years, these models have been studied and improved in order to deal with non-homogeneous frameworks. In particular, we can find in the literature some heterogeneous GDM models at three different levels: i) heterogeneity at the preference representation structure level (orders, utility functions or preference relations) [15[16], ii) heterogeneity at the preference representation domain level (numeric, linguistic, multigranular, interval numbers) [17/18 1920] and iii) heterogeneity at the importance degree of experts and criteria level [21/22].

On this third studied heterogeneity level, the importance degrees associated to the experts are fixed through the problem. However, there are some situations in which experts have an heterogeneous knowledge of the problem environment. Thus, their importance degrees can not be associated regardless what is being evaluated and it should be different for each criterion. Therefore, it is still necessary to study and improve the existing heterogeneous GDM models.

Accordingly, we propose to tackle heterogeneous MCGDM problem based on nonhomogeneous frameworks with heterogeneously specialized experts' preferences. To do so, we assume that experts give their assessments about the alternatives in natural language, using preference relations [15] as the preferences representation structure on a fuzzy linguistic domain. For this reason, we use a fuzzy linguistic modelling [4] to represent the experts' opinions. This kind of modelling is an approximate technique which represents qualitative aspects as linguistic values by means of linguistic variables [23], that is, variables whose values are not numbers but words or sentences in a natural or artificial language. To compute the quality assessments we use computing with words tools based on linguistic aggregation operators.

The aim of this paper is to present a new model of MCGDM selection process based on heterogeneously specialized experts' preferences, where the set of experts is established depending on the different criteria of the problem. Moreover, an expert's opinion 
will have different importance level according to the criterion which is taken into account and the assessed alternatives.

In order to do this, the paper is set out as follows. Preliminaries are presented in Section 2. Section 3 defines the new fuzzy linguistic MCGDM selection process based on heterogeneously specialized experts' preferences and finally, Section 4 draws our conclusions.

\section{Preliminaries}

In this section we present some considerations about MCGDM problems, heterogeneity in group decision making and the basis of a fuzzy linguistic approach.

\subsection{MCGDM Problems}

In a GDM problem we have a finite set of feasible alternatives. $X=\left\{x_{1}, x_{2}, \ldots\right.$ ,$\left.x_{n}\right\},(n \geq 2)$ and the best alternatives from $X$ have to be identified using the information given by a set of experts, $E=\left\{e_{1}, e_{2}, \ldots, e_{m}\right\},(m \geq 2)$, according to a set of criteria $C=\left\{c_{1}, c_{2}, \ldots, c_{p}\right\},(p \geq 2)$.

Resolution methods for GDM problems are usually composed by two different processes [4] (see Figure 1):

1. Consensus process: Clearly, in any decision process, it is preferable that the experts reach a high degree of consensus on the solution set of alternatives. Thus, this process refers to how to obtain the maximum degree of consensus or agreement among the experts on the solution alternatives.

2. Selection process: This process consists in how to obtain the solution set of alternatives from the opinions on the alternatives given by the experts. Furthermore, the selection process is composed of two different phases:

(a) Aggregation phase: This phase uses an aggregation operator in order to transform the individual preferences on the alternatives into a collective preference.

(b) Exploitation phase: This phase uses choice functions [24] in order to transform the collective preference into a partial ranking of alternatives that helps to make the final decision.

In this paper, we center our attention only in the selection process, where the experts will provide their preferences about the set of alternatives on each criteria by using words in natural language by means of the fuzzy linguistic approach and a ranking of alternatives is obtained according to experts' preferences.

\subsection{Heterogeneity in Group Decision Making}

Recently, several authors have studied and approached MCGDM problems from different angles, showing that this kind of problems are not always homogeneous. We can classify them into three different heterogeneity levels. 


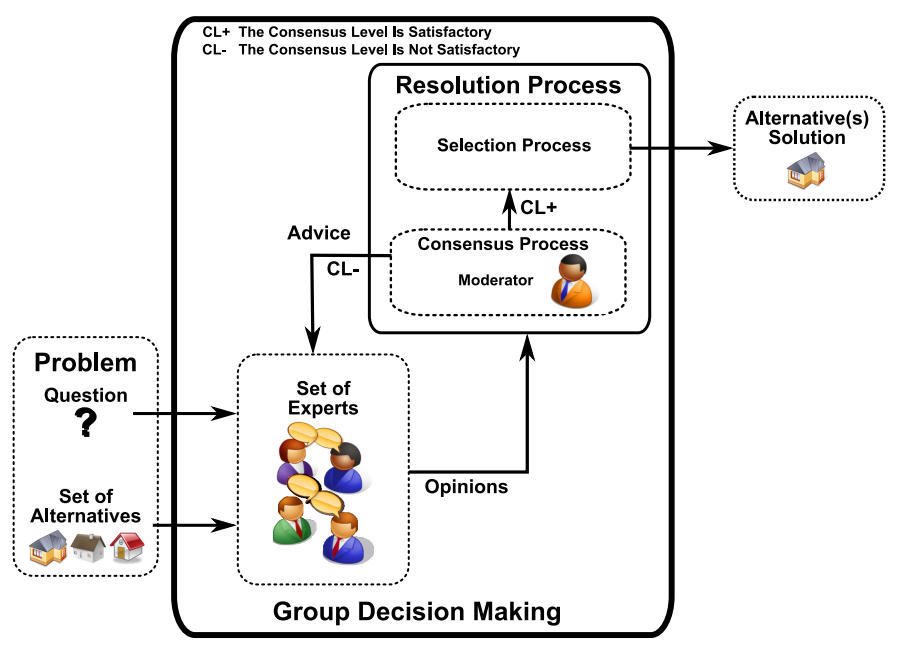

Fig. 1. Resolution process of a GDM problem

1. The first heterogeneity level studied in the literature $[15[16|25| 26]$, is focused on the preference representation structures. Usually, each expert $e_{h}$ provides his/her preferences on the alternatives $X=\left\{x_{1}, x_{2}, \ldots, x_{n}\right\}$, separately according to each criteria $C=\left\{c_{1}, c_{2}, \ldots, c_{p}\right\}$ by means of different preference's representation format, the most commonly used are:

- Preference orderings of alternatives: $O^{k}=\left\{o^{k}(1), \ldots, o^{k}(n)\right\}$, where $o^{k}(\cdot)$ is a permutation function over the index set, $\{1, \ldots, n\}$, for the expert, $e_{k}$, defining an ordered vector of alternatives, from best to worst.

- Utility functions: $U^{k}=\left\{u_{1}^{k}, \ldots, u_{n}^{k}\right\}, u_{i}^{k} \in[0,1]$, where $u_{i}^{k}$ represents the utility evaluation given by the expert $e_{k}$ to $x_{i}$.

- Fuzzy preference relations: $P^{k} \subset X \mathrm{X} X$, with a membership function, $\mu_{P^{k}}$ : $X \mathrm{X} X \rightarrow[0,1]$, where $\mu_{P^{k}}\left(x_{i}, x_{j}\right)=p_{i j}^{k}$ denotes the preference degree of $x_{i}$ over $x_{j}$.

- Multiplicative preference relations: $A^{k} \subset X \times X$, where the intensity of preference, $a_{i j}^{k}$, is measured using a ratio scale, particularly the 1/9 to 9 scale.

Fuzzy preference relations are widely used in this kind of problems because they are more informative than preference orderings or utility functions [15], allowing the comparison of the alternatives in a pair by pair basis. Thus, users have much more freedom at giving their preferences and they can gain expressivity against other preference representations. When cardinality of $X$ is small, the preference relation may be conveniently represented by an $n \times n$ matrix $P^{h s}=\left(p_{i j}^{h s}\right)$.

2. The second heterogeneity level is focused on the preference representation domain (numeric, linguistic, multi-granular, interval numbers) [17/18|19|20|26|27 28]. We propose the use of a fuzzy linguistic approach that is presented in Section 2.3

3. Finally, the third heterogeneity level [2122], deals with some classical heterogeneous decision scenarios, where every expert has an associated weight value in order to model their different importance levels or knowledge degrees. Furthermore, 
in some multi-criteria decision scenarios, we can find criteria with different weight values [12]. In these situations, the experts' or criteria's weight values are always established a priori, regardless what is being evaluated.

However, there are heterogeneous situations in which the knowledge degree of each expert is different depending on the criterion. In such a way, the opinion of an expert specialized on a certain criterion should be more important than one of another expert not familiarized with this same criterion. So, an expert could have different relevance according to what is being evaluated at every moment. Consequently, in this paper, we propose a new approach to model an experts' weights establishment mechanism in accordance with the criterion which is taken into account and the assessed alternatives.

\subsection{Fuzzy Linguistic Approach}

Several authors have provided interesting results on GDM with the help of fuzzy theory [1 5|8[17|18], but there are situations in which the information cannot be assessed precisely in a quantitative form but may be in a qualitative one. For example, when attempting to qualify phenomena related to human perception, we are often led to use words in natural language instead of numerical values. In other cases, precise quantitative information cannot be stated because either it is unavailable or the cost for its computation is too high and an "approximate value" can be applicable, eg. when evaluating the speed of a car, linguistic terms like fast, very fast or slow can be used instead of numeric values [1729]. The use of Fuzzy Sets Theory has given very good results for modelling qualitative information [23].

Fuzzy linguistic modelling is a tool based on the concept of linguistic variable [23] to deal with qualitative assessments. It has proven its usefulness in many problems, e.g., in quality evaluation, information retrieval models, decision making, and so on [30 31 32]. Ordinal fuzzy linguistic modelling [4] is a very useful kind of fuzzy linguistic approach proposed as an alternative tool to the traditional fuzzy linguistic modelling which simplifies the computing with words process as well as linguistic aspects of problems. It is defined by considering a finite and totally ordered label set $S=\left\{s_{i}\right\}, i \in\{0, \ldots, g\}$ in the usual sense, i.e., $s_{i} \geq s_{j}$ if $i \geq j$, and with odd cardinality (usually 7 or 9 labels). The mid term represents an assessment of "approximately 0.5 ", and the rest of the terms are placed symmetrically around it. The semantics of the label set is established from the ordered structure of the label set by considering that each label for the pair $\left(s_{i}, s_{g-i}\right)$ is equally informative [17]. For example, we can use the following set of seven labels to represent the linguistic information:

$$
S=\{\mathrm{N}=\text { Null, VL=Very Low, L=Low, M=Medium, H=High, VH=Very High, }
$$
$\mathrm{P}=$ Perfect $\}$.

In any linguistic model we also need some management operators to deal with linguistic information. An advantage of the ordinal fuzzy linguistic modeling is the simplicity and speed of its computational model. It is based on the symbolic computational model [4] and acts by direct computation on labels by taking into account the order of such linguistic assessments in the ordered structure of labels. Usually, the ordinal fuzzy linguistic model for computing with words is defined by establishing i) a negation operator, ii) comparison operators based on the ordered structure of linguistic terms, and 
iii) adequate aggregation operators of ordinal fuzzy linguistic information. In most ordinal fuzzy linguistic approaches the negation operator is defined from the semantics associated to the linguistic terms as

$$
N E G\left(s_{i}\right)=s_{j} \mid j=(g-i)
$$

and there are defined two comparison operators of linguistic terms:

1. Maximization operator: $M A X\left(s_{i}, s_{j}\right)=s_{i}$ if $s_{i} \geq s_{j}$; and

2. Minimization operator: $\operatorname{MIN}\left(s_{i}, s_{j}\right)=s_{i}$ if $s_{i} \leq s_{j}$.

Using these operators it is possible to define automatic and symbolic aggregation operators of linguistic information, as for example the Linguistic Ordered Weighted Averaging (LOWA) operator [33]. Sometimes, the different items that we need to aggregate have as well an associated weight. It has to be taken into account on the aggregation operator selection. So, in these situations, we can use the Linguistic Induced Ordered Weighted Averaging (L-IOWA) operator that is a linguistic version of the IOWA operator [34 35 [ 36 . 36 .

Definition 1. L-IOWA operator is defined as follows [37]:

$$
\Phi_{W}\left(\left\langle u_{1}, s_{\alpha_{1}}\right\rangle, \ldots,\left\langle u_{n}, s_{\alpha_{n}}\right\rangle\right)=w_{1} \cdot s_{\gamma_{1}} \oplus w_{2} \cdot s_{\gamma_{2}} \oplus \ldots \oplus w_{n} \cdot s_{\gamma_{n}}=s_{\operatorname{round}(\bar{\gamma})}
$$

where $\bar{\gamma}=\sum_{j=1}^{n} w_{j} \cdot \gamma_{j}, w=\left(w_{1}, w_{2}, \ldots, w_{n}\right)$ is a weighting vector, such that $w_{j} \in$ $[0,1], \sum_{j=1}^{n} w_{j}=1, s_{\gamma_{j}}$ is the $s_{\alpha_{i}}$ value of the pair $\left\langle u_{i}, s_{\alpha_{i}}\right\rangle$ having the jth largest $u_{i}$, and $u_{i}$ in $\left\langle u_{i}, s_{\alpha_{i}}\right\rangle$ is referred to as the order inducing variable and $s_{i}$ as the linguistic argument variable.

A natural question in the definition of this operator is how to obtain the associated weighting vector. In [38], an expression to obtain $\mathrm{W}$ that allows to represent the concept of fuzzy majority [39] by means of a fuzzy linguistic non-decreasing quantifier Q [40] was defined:

$$
w_{i}=Q(i / n)-Q((i-1) / n), i=1, \ldots, n .
$$

\section{A New Fuzzy Linguistic MCGDM Model Based on Heterogeneous Experts' Opinions}

In this section we present a new approach to deal with MCGDM problems in which the main characteristic is the heterogeneous knowledge level of each expert among the multiple criteria. Usually, experts become to reach an excessive specialization on some specific aspects of their own field (heterogeneously specialized experts). So, each of them has a different knowledge level that depends on the criterion that is being taken into account to assess the alternatives. This characteristic is important for the problem management and has to be taken into account not only on the choice of experts, but also on the combination of their individual preferences in order to obtain a more realistic and appropriate collective preference on each criterion.

Consequently, we propose a new computation model composed of two different steps: 
1. To obtain an appropriate set of heterogeneously specialized experts and their preferences.

2. To compute the ranking of alternatives through a fuzzy linguistic MCGDM selection process based on heterogeneously specialized experts' preferences.

\subsection{Obtaining the Appropriate Set of Heterogeneously Specialized Experts and Their Preferences}

When the field of the decision is large and non homogeneous, there are multiple criteria and different kinds of experts together in the problem framework. Therefore, the choice of experts becomes an important task. To do so in an appropriate way, we need to know the experts' typology or the kind of specialization of each expert before starting the decision making process. This requirement is necessary in order to select experts to cover every criterion with knowledge enough. For example, in library evaluation, to fully evaluate the quality of an university library, it is necessary to collect not only students' opinions who just use study resources but also researchers' preferences who usually are focused on research resources or staff's opinions who knows much better the quality of the space resources.

In these situations, it is necessary an initial approach to the problem in order to get the different alternatives and criteria. Once we know this information and the different specialization of each expert we can start the choice of experts step. In this way, we propose to select a suitable set of experts with heterogeneous knowledge enough, covering all the decision criteria, from any expert database. To do so, it is quite important the figure of the moderator, who is a person that has a deep knowledge about the problem (alternatives and criteria), and he is able to select a suitable and balanced set of experts.

For example, in order to select the best university library from a set of them for a particular use (to study for an exam, to research on a new topic...) it is clear that the alternatives are the different libraries of the university and the criteria to evaluate them are the resources offered by each one (space resources, electronic resources, paper resources or human resources).

At this point, is the moderator who select $m$ experts of an experts' database that previously agree to take part in this kind of studies. To have a suitable group of experts, the set has to be balanced, that is, it seems reasonable to have the same number of experts of each kind. Thus, in our example, the set will be composed of $z$ students, $z$ researchers and $z$ staff members in order to have a thorough collective knowledge of every criterion.

In addition, to be fair and correctly manage the heterogeneous collective knowledge, we propose that the opinion of an expert specialized on a certain criterion is more important than one of another expert not familiarized with this same criterion. In such a way, the weight values not only depend on the experts, but also on the criterion followed by the expert to assess the alternatives. Particularly, in academic libraries, this situation is frequently presented because students, researchers and staff members use the library in a different way, according to their own purposes. For instance, a student knows the main drawbacks and advantages of the space resources much better than a researcher because he is using it everyday while researchers usually work in their own offices. 
On the other hand, a researcher has a more deep insight on the quality level of research resources like international journals or database access than a standard student.

To model this situation it is necessary to properly define the above mentioned experts' typologies and with them to define the importance of the experts' opinions on the alternatives over each criterion. The moderator is responsible for the weights refinement in each particular case by assigning an expert's weight value to each kind of experts for each single criterion ecw.

Finally, the last moderator's task is to assign the relevance of each criterion for the particular problem by mean of some importance values. In this way, if the library selection is performed with the aim of studying for an exam, the space resources is the most important criterion but if the use of the library will be to research on new technologies the library side lose relevance being more important the electronic resources. These importance values will be treated later as criteria's weight values $c w$ by the aggregation operator.

The ordinal fuzzy linguistic modeling approach lets use a set of linguistic labels as weight values instead of numbers. So, these weights $e c w$ and $c w$ can be expressed using any set of linguistic labels. A feasible set of $l$ labels could be the next: $S=\left\{s_{1}=\right.$ VeryLow, $s_{2}=$ Low, $s_{3}=$ Medium, $s_{4}=$ High, $s_{5}=$ VeryHigh $\}$.

Once we have selected the most suitable sets of alternatives, criteria and experts, the decision making process starts with the collection of every expert's opinion. Thus, each expert must give his own assessments on every alternative for each criterion.

We assume that each expert $e_{h}$ provides his/her preferences $\left\{P^{h 1}, P^{h 2}, \ldots, P^{h p}\right\}$ by means of $p$ fuzzy linguistic preference relations (FLPR) characterized by a membership function [4]:

$$
\mu_{P}: X \times X \longrightarrow S
$$

where $S$ is a set of linguistic labels and $p$ is the number of criteria.

For instance, by using the set of seven labels introduced in Section 2, an expert $e_{h}$ could provide the following FLPR on a set of four alternatives according to the criterion $c_{s}$.

$$
P^{h s}=\left(\begin{array}{cccc}
- & N & H & M \\
P & - & L & M \\
L & H & - & V L \\
M & M & V H & -
\end{array}\right)
$$

According to $p_{24}^{h s}=M$ and $p_{21}^{h s}=P, e_{h}$ considers that on the criterion $c_{s}$, alternatives $x_{2}$ and $x_{4}$ are at the same level but $x_{2}$ is better than $x_{1}$ respectively.

\subsection{Fuzzy Linguistic MCGDM Selection Process with Heterogeneously Specialized Experts}

In order to obtain a collective assessment from the whole group of experts, the individual opinions have to be computed using an aggregation operator.

When each expert has provided all his preferences (FLPRs) on the alternatives for each criterion, we can obtain a ranking of them by applying a selection process [14]. The selection process consists of two different phases: 


\section{Aggregation of individual heterogeneously specialized experts' FLPRs on mul- tiple criteria:}

The aggregation phase defines a collective preference relation, $P^{c}=\left(p_{i j}^{c}\right)$, obtained by means of the aggregation of all individual linguistic preference relations $\left\{P^{11}, P^{12}, \ldots, P^{1 p}, P^{21}, P^{22}, \ldots, P^{2 p}, \ldots, P^{m p}\right\}$. It indicates the global preference among every pair of alternatives according to all the experts' opinions taking into account the whole set of criteria.

To To deal with this situations, we propose a fuzzy linguistic MCGDM aggregation process with two different phases:i) aggregation of individual FLPRs on each criterion and ii) aggregation of collective FLPRs on each criterion.

(a) Aggregation of individual FLPRs on each criterion:

At this point, in order to aggregate the individual preferences taking into account every criteria and the heterogeneous knowledge degrees, the first step is to obtain a collective preference relation over each criterion $c_{s}, P^{c s}=\left(p_{i j}^{c s}\right)$, obtained by means of the aggregation of all individual linguistic preference relations $\left\{P^{1 s}, P^{2 s}, \ldots, P^{m s}\right\}$. It indicates the global preference among every pair of alternatives according to the criterion $c_{s}$.

Thus, to compute each collective fuzzy linguistic preference degree $P^{c s}$ according to the knowledge level of each expert, we propose to use the L-IOWA operator with the linguistic experts' weight values $e c w$ as the values of the order inducing variable, i.e.,

$$
p_{i j}^{c s}=\Phi_{W}\left(\left\langle e c w^{1 s}, p_{i j}^{1 s}\right\rangle, \ldots,\left\langle e c w^{m s}, p_{i j}^{m s}\right\rangle\right)
$$

(b) Aggregation of collective FPLRs on each criterion:

Once all the individual FLPRs $P^{h s}$ have been aggregated obtaining a collective FLPR $P^{c s}$ for each criterion, this second aggregation step defines a collective preference relation, $P^{c}=\left(p_{i j}^{c}\right)$, computed by means of the aggregation of all collective FLPRs obtained in the previous step $\left\{P^{c 1}, P^{c 2}, \ldots, P^{c p}\right\}$. It indicates the global preference among every pair of alternatives according to all of different criteria.

The aggregation operator of this step depends on the importance of the criteria, therefore, we propose to use again the L-IOWA operator with the linguistic criteria's weight values $c w$ as the values of the order inducing variable, i.e.,

$$
p_{i j}^{c}=\Phi_{W}\left(\left\langle c w^{1}, p_{i j}^{c 1}\right\rangle, \ldots,\left\langle c w^{p}, p_{i j}^{c p}\right\rangle\right)
$$

\section{Exploitation of the collective FLPR:}

This phase transforms the global information about the alternatives, $P^{c}$, into a global ranking of them. In such a way, the set of solution alternatives is obtained. The global ranking is obtained applying these two choice degrees of alternatives on the collective preference relation QGDD and QGNDD. These degrees can be studies in more detail in [24]:

\section{Conclusions}

In this paper, we have presented a new approach, to deal with MCGDM problems, in which the main contribution is the possibility of join with more accuracy heterogeneously specialized experts' opinions. It has sense when each expert has different 
knowledge level among the different aspects of the discussion field. To do so, we propose a balanced selection of experts, the use of FLPRs as format of preferences representation and the use of a proper aggregation operator to model the heterogeneity among experts. Using this model, the heterogeneous knowledge of the different kinds of expert is managed with more accuracy over each particular situation instead of doing it over the whole problem. In such a way, better results and decisions can be obtained.

In the future, we will use incomplete information models and mobile technologies in order to present a dynamic decision making process in which the different elements of the problem (alternatives, experts, weights and so on) can be changed through the time.

Acknowledgements. This paper has been developed with the financing of FEDER funds in FUZZYLING Project TIN200761079, FUZZYLING-II Project TIN201017876, PETRI Project PET20070460, Andalusian Excellence Project TIC-05299, and project of Ministry of Public Works 90/07.

\section{References}

1. Herrera, F., Herrera-Viedma, E., Verdegay, J.: A sequential selection process in group decision making with a linguistic assessment approach. Information Sciences 85(4), 223-239 (1995)

2. Herrera, F., Herrera-Viedma, E., Verdegay, J.: A model of consensus in group decision making under linguistic assessments. Fuzzy Sets and Systems 78(1), 73-87 (1996)

3. Herrera, F., Herrera-Viedma, E., Verdegay, J.: Linguistic measures based on fuzzy coincidence for reaching consensus in group decision making. International Journal of Approximate Reasoning 16, 309-334 (1997)

4. Herrera, F., Herrera-Viedma, E.: Linguistic decision analisis: steps for solving decision problems under linguistic information. Fuzzy Set and Systems 115, 67-82 (2000)

5. Kacprzyk, J., Fedrizzi, M.: Multiperson decision making models using fuzzy sets and possibility theory. Kluwer Academic Publishers, Dordrecht (1990)

6. Triantaphyllou, E.: Multi-criteria decision making methods: a comparative study. Kluwer Academic Publishers, Dordrecht (2000)

7. Choi, D.H., Ahn, B., Kim, S.: Multicriteria group decision making under incomplete preference judgments: using fuzzy logic with a linguistic quantifier. International Journal of Intelligent Systems 22(6), 641-660 (2007)

8. Fodors, J., Roubens, M.: Fuzzy preference modelling and multicriteria decision support. Kluwer Academic Publishers, Dordrecht (1994)

9. Fu, G.: A fuzzy optimization method for multicriteria decision making: an application to reservoir flood control operation. Expert Systems with Applications 31(1), 145-149 (2008)

10. Gheorghe, R.A., Bufardi, A., Xirouchakis, P.: Fuzzy multicriteria decision aid method for conceptual design. Cirp Annals-Manufacturing Technology 54(1), 152-154 (2005)

11. Lu, J., Zhang, G., Ruan, D.: Intelligent multi-criteria fuzzy group decision making for situation assessments. Soft. Computing 12(3), 289-299 (2008)

12. Lu, J., Zhu, Y., Zeng, X., Ma, J., Zhang, G.: A linguistic multi-criteria group decision support system for fabric hand evaluation. Fuzzy Optimization and Decision Making 8(4), 395-413 (2009)

13. Ma, J., Lu, J., Zhang, G.: Decider: A fuzzy multi-criteria group decision support system. Knowledge-Based Systems 23(1), 23-31 (2010)

14. Yager, R.: Non-numeric multi-criteria multi-person decision making. Group Decision and Negotiation 2, 81-93 (1993) 
15. Chiclana, F., Herrera, F., Herrera-Viedma, E.: Integrating three representation models in fuzzy multipurpose decision making based on fuzzy preference relations. Fuzzy Sets and Systems 97(1), 33-48 (1998)

16. Chiclana, F., Herrera, F., Herrera-Viedma, E.: Integrating multiplicative preference relations in a multiplicative decision making model based on fuzzy preference relations. Fuzzy Sets and Systems 122(2), 277-291 (2001)

17. Alonso, S., Cabrerizo, F., Chiclana, F., Herrera, F., Herrera-Viedma, E.: Group decisionmaking with incomplete fuzzy linguistic preference relations. International Journal of Intelligent Systems 24(2), 201-222 (2009)

18. Cabrerizo, F., Alonso, S., Herrera-Viedma, E.: A consensus model for group decision making problems with unbalanced fuzzy linguistic information. International Journal of Information Technology \& Decision Making 8(1), 109-131 (2009)

19. Herrera, F., Herrera-Viedma, E., Martínez, L.: A fuzzy linguistic methodology to deal with unbalanced linguistic term sets. IEEE Transactions on Fuzzy Systems 16(2), 354-370 (2008)

20. Mata, F., Martínez, L., Herrera-Viedma, E.: An adaptive consensus support model for group decision making problems in a multi-granular fuzzy linguistic context. IEEE Transactions on Fuzzy Systems 17(2), 279-290 (2009)

21. Kacprzyk, J., Zadrozny, S., Ras, Z.: Action rules in consensus reaching process support. In: 9th International Conference on Intelligent Systems Design and Applications (ISDA 2009), pp. 809-814 (2009)

22. Perez, I.J., Cabrerizo, F., Herrera-Viedma, E.: A mobile decision support system for dynamic group decision making problems. IEEE Transactions on Systems, Man and Cybernetics - Part A: Systems and Humans 40(6), 1244-1256 (2010)

23. Zadeh, L.: The concept of a linguistic variable and its applications to approximate reasoning. Information Sciences, Part I, II, III 8, 8, 9, 199-249, 301-357, 43-80 (1975)

24. Herrera, F., Herrera-Viedma, E.: Choice functions and mechanisms for linguistic preference relations. European Journal of Operational Research 120, 144-161 (2000)

25. Xu, Z.: Multiple-attribute group decision making with different formats of preference information on attributes. IEEE Transactions on Systems, Man and Cybernetics Part BCybernetics 37(6), 1500-1511 (2007)

26. Xu, Z., Chen, J.: Magdm linear-programming models with distinct uncertain preference structures. IEEE Transactions on Systems, Man and Cybernetics Part B-Cybernetics 38(5), 1356-1370 (2008)

27. $\mathrm{Xu}, \mathrm{Z}$.: A method based on the dynamic weighted geometric aggregation operator for dynamic hybrid multi-attribute group decision making. International Journal of Uncertainty, Fuzziness and Knowledge-Based Systems 17(1), 15-33 (2009)

28. $\mathrm{Xu}, \mathrm{Z}$.: An interactive approach to multiple attribute group decision making with multigranular uncertain linguistic information. Group Decision and Negotiation 18, 119-145 (2009)

29. Herrera, F., Alonso, S., Chiclana, F., Herrera-Viedma, E.: Computing with words in decision making: Foundations, trends and prospects. Fuzzy Optimization and Decision Making 8(4), 337-364 (2009)

30. García-Lapresta, J., Meneses, L.: Modelling rationality in a linguistic framework. Fuzzy Sets and Systems 160, 3211-3223 (2009)

31. Herrera-Viedma, E., Pasi, G., López-Herrera, A.G., Porcel, C.: Evaluating the information quality of web sites: A methodology based on fuzzy. Journal of the American Society for Information Science and Technology 57(4), 538-549 (2006)

32. Porcel, C., Moreno, J., Herrera-Viedma, E.: A multi-disciplinar recommender system to advice research resources in university digital libraries. Expert Systems with Applications 36(10), 12520-12528 (2009)

33. Herrera, F., Herrera-Viedma, E., Verdegay, J.: Direct approach processes in group decision making using linguistic owa operators. Fuzzy Sets and Systems 79, 175-190 (1996) 
34. Chiclana, F., Herrera-Viedma, E., Herrera, F., Alonso, S.: Some induced ordered weighted averaging operators and their use for solving group decision-making problems based on fuzzy preference relations. European Journal of Operational Research 182(1), 383-399 (2007)

35. Yager, R.: Induced aggregation operators. Fuzzy Sets and Systems 137(1), 59-69 (2003)

36. Yager, R., Filev, D.: Induced ordered weighted averaging operators. IEEE Transactions on Systems, Man, and Cybernetics 29(2), 141-150 (1999)

37. $\mathrm{Xu}, \mathrm{Z}$.: Eowa and eowg operators for aggregating linguistic labels based on linguistic preference relations. International Journal of Uncertainty, Fuzziness and Knowledge-Based Systems 12, 791-810 (2004)

38. Yager, R.: On ordered weighted averaging aggregation operators in multicriteria decision making. IEEE Transactions on Systems, Man, and Cybernetics 18(1), 183-190 (1988)

39. Kacprzyk, J.: Group decision making with a fuzzy linguistic majority. Fuzzy Sets and Systems 18, 105-118 (1986)

40. Zadeh, L.: A computational approach to fuzzy quantifiers in natural languages. Computer and Mathematics with Applications 9(1), 149-184 (1983) 